\title{
Radioprotection associée aux nouvelles évolutions, diagnostiques et thérapeutiques, en médecine nucléaire
}

\author{
B. AUBERT ${ }^{1}$, J.-F. CHATAL ${ }^{2}$
}

(Manuscrit reçu le 17 mai 2005, accepté le 25 octobre 2005)

RÉSUMÉ Une véritable révolution technologique a, au cours des 5 dernières années, profondément modifié le périmètre d'application et les perspectives de la médecine nucléaire, en particulier de la cancérologie nucléaire. Dans le cadre des applications diagnostiques, la tomographie par émission de positons (TEP) avec $\mathrm{le}^{18} \mathrm{~F}$-fluoro déoxyglucose (FDG) a eu un impact déterminant sur la stratégie diagnostique des oncologues en ajoutant des informations fonctionnelles très précieuses aux informations morphologiques de l'imagerie conventionnelle. De nombreux autres traceurs fluorés, en cours d'évaluation clinique, sont destinés à étudier des fonctions tumorales diverses (prolifération tumorale, hypoxie, apoptose chimioinduite, etc.) pouvant avoir des répercussions thérapeutiques importantes. D'autres émetteurs de positons seront disponibles à terme, comme le ${ }^{64} \mathrm{Cu}$ (période de 12 heures), le ${ }^{124} \mathrm{I}$ (période de 4,1 jours) et le ${ }^{86} \mathrm{Y}$ (période de 15 heures) dans des indications comme l'immuno-TEP ou la dosimétrie pré-thérapeutique qui ne peuvent être développées avec le ${ }^{18} \mathrm{~F}$ du fait de sa période trop courte. Dans le cadre des applications thérapeutiques, la radiothérapie interne, longtemps limitée au cancer thyroïdien différencié, s'est étendue à beaucoup d'autres types de cancers du fait de la disponibilité de nouveaux vecteurs de radionucléides comme les anticorps monoclonaux (radioimmunothérapie) ou des peptides (radiopeptide-thérapie), de nouvelles méthodes de ciblage tumoral et de nouveaux radionucléides, en particulier des émetteurs de particules alpha (alpha-thérapie). Toutes ces évolutions technologiques ont bien sûr une implication en radioprotection en ce qui concerne l'exposition du personnel de médecine nucléaire, de l'entourage familial proche et plus généralement de l'environnement. Actuellement l'essentiel des dispositions règlementaires s'applique au ${ }^{99 m} \mathrm{Tc}$ pour les applications diagnostiques et au ${ }^{131}$ I en thérapie, elles ont été étendues en 2001 au ${ }^{18} \mathrm{~F}$ dans le cadre des contraintes de radioprotection liées à l'utilisation de FDG. Concernant l'exposition de l'entourage proche du malade, la règlementation est actuellement limitée au seuil d'activité de $740 \mathrm{MBq}$ de ${ }^{131}$ I qui impose un confinement hospitalier du malade. Une indication indirecte de cette exposition peut être donnée par le débit d'exposition $(\mathrm{en} \mu \mathrm{Sv} / \mathrm{h})$ à 1 mètre pour une source de $1 \mathrm{MBq}$. Si on prend en compte la valeur du débit et la période physique, il apparaît, qu'à activité identique, $\mathrm{le}^{52} \mathrm{Fe}, \mathrm{le}^{86} \mathrm{Y}$ et le ${ }^{124} \mathrm{I}$ sont des radionucléides émetteurs de positons qui présentent un débit élevé à la sortie du service et pendant les heures qui suivent l'injection. Concernant l'exposition de l'environnement, la règlementation actuelle impose une concentration radioactive, à l'émissaire de l'établissement, de $1000 \mathrm{~Bq} / \mathrm{L}$ pour $\mathrm{le}^{99 \mathrm{~m}} \mathrm{Tc}$ et de $100 \mathrm{~Bq} / \mathrm{L}$ pour le ${ }^{131} \mathrm{I}$. Pour les nouveaux radionucléides, d'autres hypothèses que celle d'une concentration

IRSN/DRPH/SER/Unité d'expertise en radioprotection médicale, BP 17, 92262 Fontenay-aux-Roses Cedex, France.

2 UMR INSERM U 601, Université de Nantes, 44093 Nantes Cedex, France. 
permanente de $100 \mathrm{~Bq} / \mathrm{L}$ doivent être retenues et des études d'impact, similaires à celles pratiquées dans le domaine du nucléaire devraient être effectuées.

ABSTRACT Radiation protection for innovative diagnostic and therapeutic approaches in nuclear medicine.

A real technological revolution has deeply modified the field of application and perspectives of nuclear medicine, and nuclear oncology in particular, during the past 5 years. Diagnostic applications such as positron emission tomography (PET) with ${ }^{18}$ F-fluorodeoxyglucose (FDG) have had a significant impact on the diagnostic strategy adopted by medical oncologists, with the addition of invaluable functional data to already available anatomical data provided by conventional imaging modalities. Numerous other ${ }^{18} \mathrm{~F}$-labeled tracers currently under clinical evaluation have been developed to study various tumor functions (tumor proliferation, hypoxia, chemotherapy-induced apoptosis, etc.). These tracers may have a considerable impact on therapeutic strategies. Other positron-emitting radionuclides, such as copper-64, iodine-124, and yttrium-86 (whose respective half-lives are 12.7 hours, 4.2 days, and 14.7 hours) will soon be available for certain clinical indications, such as immuno-PET (with monoclonal antibodies or antibody fragments used as carriers) or pretreatment dosimetry, which cannot be performed with fluorine-18 due its short half-life. As far as therapeutic applications are concerned, the use of internal radiotherapy, which has been restricted to thyroid cancer for a long time, was recently extended to other cancers as new carriers, such as monoclonal antibodies (radioimmunotherapy) or peptides (radiopeptide therapy), new targeting methods (pretargeting), and new radionuclides, especially alpha particle emitters (alpha therapy), became available. These technological advances require that specific radiation safety regulations be implemented to protect nuclear medicine personnel, patients' close relatives, and the environment. Most current regulations concern diagnostic applications with technetium-99m and therapeutic applications with iodine-131. Regulations pertaining to the clinical use of ${ }^{18} \mathrm{~F}$-FDG were recently enacted (2001). Regarding exposure nuclear medicine personnel, the amount of radioactivity used for therapeutic purposes is currently limited to $740 \mathrm{MBq}$, which requires that the patient be kept in a shielded room. Radiation exposure can be roughly estimated by measuring the exposure rate (in $\mu \mathrm{Sv} / \mathrm{h}$ ) delivered at a distance of 1 meter from a source of one $\mathrm{MBq}$. When considering both the exposure rate and half-life, it appears that similar doses of iron-52, yttrium-86, and iodine-124 deliver a high dose rate immediately after injection and during the following few hours. Current regulations on environmental exposure limit radioactivity levels in hospital sewage outfalls to $1000 \mathrm{~Bq} / \mathrm{L}$, for technetium $99 \mathrm{~m}$, and $100 \mathrm{~Bq} / \mathrm{L}$, for iodine-131. Forthcoming regulations for other radionuclides should not be established solely based on general rules such as the $100 \mathrm{~Bq} / \mathrm{L}$ limit. Impact studies similar to those required in the nuclear industry, should be conducted.

Key words: nuclear medicine / radiation protection / radioimmunotherapy / radiopeptide therapy / alpha therapy

Une véritable révolution a, au cours des cinq dernières années, profondément modifié le périmètre d'application et les perspectives de la médecine nucléaire. Cette révolution concerne actuellement et principalement la cancérologie mais elle s'étendra progressivement aux autres spécialités en particulier la cardiologie et la neurologie. Elle se traduit, pour les applications diagnostiques, par le rapide développement de la tomographie par emission de positons (TEP), le plus souvent 
associée à la tomodensitométrie (systèmes hybrides TEP/scanner) et, pour les applications thérapeutiques, par le développement, également rapide de la radiopeptide thérapie et de la radioimmunothérapie. Ces développements induisent des problèmes particuliers de radioprotection qu'il convient de prendre en compte pour un bénéfice optimal de ces innovations diagnostiques et thérapeutiques.

\section{1. Évolutions diagnostiques et thérapeutiques}

Ces évolutions peuvent être classées en 2 groupes, celles concernant les applications diagnostiques qui se développent autour de la TEP et, celles liées au développement d'applications thérapeutiques.

\subsection{La tomographie par émission de positons (TEP)}

Si la TEP s'est développée ces dernières années autour du ${ }^{18} \mathrm{~F}$-fluorodeoxyglucose ([ $\left.\left.{ }^{18} \mathrm{~F}\right]-\mathrm{FDG}\right)$, il est à prévoir pour l'avenir des évolutions autour de traceurs fluorés ou utilisant d'autres émetteurs de positons.

\subsubsection{Apport diagnostique déterminant du $\left[^{18} F\right]-F D G$}

En France, le développement de cette nouvelle technique d'imagerie fonctionnelle en cancérologie de routine date du début des années 2000 avec l'installation progressive de systèmes hybrides TEP/Scanner qui permettent de fusionner les images fonctionnelles de la TEP et les images anatomiques du scanner pour un diagnostic optimal. Cette installation a été accompagnée de celle de cyclotrons de faible énergie pour la production de fluor-18 et sa distribution locale, dans un périmètre maximal de $250 \mathrm{~km}$ du fait de la courte période physique du fluor-18 (110 minutes).

Actuellement le seul agent radiopharmaceutique disponible en routine est le $\left[{ }^{18} \mathrm{~F}\right]$-FDG. Parallèlement à l'installation de nouveaux systèmes TEP/scanner, la demande d'examens par les oncologues est en progression impressionnante $(10 \%$ par mois) et les services les plus actifs pratiquent plus de 10 examens par jour. L'activité injectée en $\left[{ }^{18} \mathrm{~F}\right]-\mathrm{FDG}$ est de 5 à $8 \mathrm{MBq} / \mathrm{kg}$ soit 350 à $600 \mathrm{MBq}$ pour un patient de $70 / 75 \mathrm{~kg}$.

Un développement aussi rapide est justifié par le fort impact diagnostique de cette nouvelle imagerie sur la stratégie thérapeutique. Dans le bilan d'extension initial de plusieurs types de cancers relativement fréquents (cancers du poumon, du colon, lymphomes malins, etc.) la TEP avec FDG apporte des informations additionnelles et complémentaires de celles de l'imagerie conventionnelle 
(échographie, scanner, IRM) avec un impact important sur la décision thérapeutique (par exemple décision d'effectuer ou non une chirurgie d'exérèse lourde).

L'apport fonctionnel de la TEP avec FDG (indication de la nature maligne ou non d'une lésion) par rapport à l'information morphologique de l'imagerie conventionnelle est illustré par la situation clinique d'une masse résiduelle après chimiothérapie d'un lymphome non hodgkinien (pathologie cancéreuse en constante augmentation de fréquence). L'imagerie conventionnelle montre la persistance d'une masse anormale sans pouvoir préciser s'il s'agit d'une tumeur encore active qui nécessite alors la poursuite d'un traitement ou d'une masse fibreuse inactive qui témoigne de l'efficacité de la chimiothérapie. La TEP avec FDG permet d'apporter cette information précieuse pour le médecin oncologue.

Une autre indication non encore complètement validée de la TEP avec FDG est de prédire précocement l'efficacité d'un traitement (chimiothérapie ou radiothérapie). Actuellement la seule information dont on dispose est de nature morphologique avec un examen par scanner réalisé à la fin du traitement (augmentation, diminution ou stabilité du volume tumoral). La TEP avec FDG devrait confirmer sa capacité à prédire l'efficacité du traitement (diminution ou non de la radioactivité tumorale) très tôt après sa mise en œuvre avec la conséquence déterminante de poursuivre le traitement en cas d'efficacité démontrée (diminution de la captation tumorale de FDG) ou de l'arrêter et le remplacer par un traitement alternatif en cas d'inefficacité (absence de diminution voire augmentation de la captation tumorale).

\subsubsection{Perspectives de développement des traceurs fluorés et autres radionucléides émetteurs de positons}

La tendance actuelle à la multiplication des demandes d'examen par TEP-FDG, liées à la qualité des informations diagnostiques obtenues, ne va pas s'arrêter car le nombre de systèmes TEP-Scanner installés va continuer de croître, de nouveaux traceurs fluorés vont être introduits en clinique et plus tard de nouveaux radionucléides émetteurs de positons vont devenir disponibles tout en compliquant la radioprotection du fait de certaines de leurs caractéristiques physiques défavorables comme la période ou l'émission de rayonnement gamma énergétique associé.

\section{(a) Traceurs fluorés}

De nombreux traceurs fluorés sont en cours d'évaluation clinique et sont destinés à étudier des fonctions tumorales diverses (prolifération tumorale, hypoxie, 
apoptose chimioinduite, etc.) pouvant avoir des répercussions thérapeutiques importantes. Il est possible que le premier d'entre eux à être utilisé en routine soit la ${ }^{18}$ F-fluorothymidine (FLT) qui fait actuellement l'objet d'un projet d'étude clinique inter-cancéropôle en France. Ce traceur permet d'évaluer in vivo la cinétique de prolifération tumorale et donc l'agressivité relative d'une tumeur (Couturier et al., 2004). Il pourrait alors également prédire l'efficacité d'un traitement en indiquant son impact sur la vitesse de prolifération de la tumeur. Par rapport au FDG qui est également capté par des lésions inflammatoires bénignes compliquant ainsi l'interprétation des images (faux positifs), la FLT est peu capté par ces lésions inflammatoires, ce qui constitue un avantage important.

D'autres traceurs fluorés sont également en cours d'étude clinique mais il faudra quelques années pour évaluer leur impact clinique respectif par rapport au FDG.

\section{(b) Radionucléides émetteurs de positons}

Actuellement les applications de la TEP sont limitées à la cancérologie mais il est hautement probable que, demain, elles s'étendront à la cardiologie et la neurologie. En cardiologie nucléaire de très nombreux examens sont réalisés avec du thallium201 et du technétium-99m (MIBI) pour étudier la perfusion du myocarde avec des gamma caméras conventionnelles. Le rubidium-82, également analogue du potassium et émetteur de positons peut être utilisé en TEP même s'il n'est pas disponible en France. Du fait de sa très courte période physique $(75 \mathrm{~s})$, il est produit par un générateur de strontium-82-rubidium- 82 avec une période du strontium- 82 de 25 jours. Il est actuellement disponible aux USA dans 2 sociétés (Bristol Myers Squibb et Eastern Isotopes) à un coût encore excessif. La spécificité du rubidium-82 a été démontrée supérieure à celle du thallium et du technétium du fait de la correction d'atténuation plus efficace avec un émetteur de photons de $511 \mathrm{keV}$ (Machac, 2005). Sa courte période physique permet d'injecter de fortes activités $(1,85 \mathrm{GBq})$ au malade et de répéter l'examen 10 minutes plus tard pour des épreuves pharmacologiques. Dans ces conditions, la dosimétrie patient reste acceptable mais la radioprotection du personnel doit être sérieusement considérée même si le déclenchement de l'injection se fait à distance du malade. Cet examen est actuellement remboursé aux USA par les systèmes d'assurance. Il est difficile de prédire la disponibilité ou non de générateurs de rubidium-82 en France à moyen terme du fait de la difficulté de produire du strontium-82. En effet, ce dernier est produit par des réactions de spallation dans des accélérateurs linéaires de haute énergie (Los Alamos aux USA ou en Russie), ce qui explique en partie son coût de production. Il peut aussi être produit par des cyclotrons de forte énergie (40 à $70 \mathrm{MeV}$ ) mais qui sont encore peu disponibles pour sa production. 
Enfin, il est hautement vraisemblable que d'autres radionucléides émetteurs de positons seront disponibles à terme. Le fluor-18 est certainement le radionucléide le mieux adapté au marquage de petits vecteurs (analogues du glucose et de la thymidine) qui ont un profil pharmacocinétique court, compatible avec sa période physique (110 minutes). Par contre, il ne convient pas au marquage de gros vecteurs qui mettent plusieurs jours à se distribuer dans leurs cibles (anticorps). Certaines études précliniques d'immuno-TEP ont déjà montré l'intérêt d'utiliser des émetteurs de positons avec des périodes physiques de quelques heures à quelques jours pour le radiomarquage d'anticorps ou de « diabody » (Robinson et al., 2005). Les radionucléides les plus prometteurs sont le cuivre-64 (période de 12 heures), l'iode- 124 (période de 4,1 jours) et l'yttrium- 86 (période de 15 heures). Ces 2 derniers radionucléides posent néanmoins des problèmes de radioprotection $\mathrm{du}$ personnel encore plus importants du fait principalement d'un pourcentage d'émission non négligeable de photons gamma de haute énergie, venant s'ajouter au rayonnement photonique de $511 \mathrm{keV}$. Un questionnaire a été distribué aux USA, aux services de médecine nucléaire pour enquêter sur les souhaits prioritaires de nouveaux émetteurs de positons. L'iode-124 (période : 4,18 jours) est le plus demandé.

Au total il importe de clairement définir les règles de radioprotection qui devront s'appliquer à l'utilisation des radionucléides émetteurs de positons incluant bien sûr le fluor-18 mais s'étendant aux autres radionucléides qui pourraient devenir disponibles en France à moyen terme.

\subsection{La radiothérapie interne}

La révolution technologique en médecine nucléaire concerne aussi les applications thérapeutiques. Pendant longtemps (plusieurs décennies) la radiothérapie interne a été limitée au cancer thyroïdien avec l'iode-131 et à quelques indications orphelines faute de pouvoir disposer de vecteurs et de radionucléides adéquats. Depuis une dizaine d'années les vecteurs originaux se multiplient avec en particulier des anticorps monoclonaux (radioimmunothérapie) (Sharkey et Goldenberg, 2005), et des peptides (radiopeptide thérapie) (Gotthardt et al., 2004). L'iode-131 est toujours utilisé mais il tend à être remplacé par l'yttrium-90, émetteur bêta pur, qui ne nécessite pas le confinement des malades en chambre spécialement aménagée pour la radioprotection. D'autres radionucléides émetteurs bêta sont également utilisés comme le lutétium-177 et peut-être dans l'avenir le cuivre-67 qui a des propriétés physiques et biologiques intéressantes.

Enfin une nouvelle classe de radionucléides est en cours d'évaluation préclinique et clinique aux USA et en Europe: il s'agit de radionucléides émetteurs de particules alpha comme le bismuth-213 et l'astate-211 qui ont de 
courtes périodes physiques (45 minutes et 7,2 heures respectivement) et l'actinium-225 et le radium-233 qui ont des périodes plus longues (10 et 11 jours). Une étude clinique de phase II a déjà commencé en Suède, Grande Bretagne et Allemagne avec du chlorure de radium-223 pour le traitement antalgique des métastases osseuses des cancers de la prostate.

En ce qui concerne les problèmes de radioprotection du personnel, un élément doit être pris en considération, à savoir l'activité injectée. Comme la radiothérapie interne entraîne une toxicité principalement hématologique, cette toxicité peut être contrôlée par l'injection de cellules souches hématopoḯtiques et on a donc tendance à augmenter significativement le niveau d'activité injectée (de l'ordre de $10 \mathrm{GBq}$ avec le ${ }^{131} \mathrm{I}$ et de $4 \mathrm{GBq}$ pour le ${ }^{90} \mathrm{Y}$ ).

Par ailleurs les indications de la radiothérapie interne vont probablement se multiplier car il est maintenant démontré que cette forme de radiothérapie agit en synergie avec la chimiothérapie et d'autres formes de biothérapies. Toutes ces évolutions technologiques ont bien sûr une implication en radioprotection. Le tableau I récapitule les caractéristiques des principaux radionucléides utilisés et susceptibles d'être utilisés dans les prochaines années.

\section{Les aspects de radioprotection}

Pendant longtemps, et au moins durant les trente dernières années, la radioprotection en médecine nucléaire a principalement été conçue autour du technétium-99m pour les applications diagnostiques et de l'iode-131 pour la thérapie. Les évolutions récentes décrites ci-dessus n'ont peu, ou pas, été prises en compte dans la réglementation à l'exception de l'aménagement des unités utilisant le FDG. Il serait cependant utile, si ce n'est indispensable, de s'interroger sur les problèmes de radioprotection spécifiques des nouveaux radionucléides tant au niveau du personnel que de l'entourage du patient et de l'environnement.

\subsection{Situation actuelle}

Le classement des services de médecine nucléaire fait référence à une classification relevant d'une ancienne nomenclature des actes médicaux. On différencie les services qui pratiquent la «thérapie anticancéreuse » (service de première classe) avec des niveaux d'activité supérieurs à $740 \mathrm{MBq}(20 \mathrm{mCi})$ d'iode-131 et ceux qui pratiquent le «diagnostic et la thérapie non anticancéreuse » (services de $2^{\mathrm{e}}$ et $3^{\mathrm{e}}$ classe) avec des niveaux d'activité inférieurs à cette valeur. Il en résulte que les patients traités avec plus de $740 \mathrm{MBq}$ d'iode131 doivent être hospitalisés alors que ceux traités avec une activité inférieure sont gérés en ambulatoire. En complément de cette mesure, on peut noter que les 
TABLEAU I

Récapitulatif des caractéristiques des principaux radionucléides utilisés ou susceptibles d'être utilisés dans les prochaines années.

Summary of features of radionuclides which will be or could be used in the coming years.

\begin{tabular}{|c|c|c|c|c|c|c|}
\hline \multirow{2}{*}{ Radionucléide } & \multirow{2}{*}{$\begin{array}{l}\text { Période } \\
\text { physique } \\
\text { (heures) }\end{array}$} & \multirow{2}{*}{$\begin{array}{l}\text { Énergie } \alpha, \beta^{-} \\
\beta^{+} \max (\mathbf{k e V})\end{array}$} & \multicolumn{2}{|c|}{ Émission gamma } & \multirow{2}{*}{$\begin{array}{l}\text { Utilisation } \\
\text { potentielle }\end{array}$} & \multirow{2}{*}{$\begin{array}{c}\text { Activité probable } \\
\text { (MBq) }\end{array}$} \\
\hline & & & $(\%)$ & $(\mathbf{k e V})$ & & \\
\hline \multicolumn{7}{|l|}{ Émetteurs $\beta^{+}$} \\
\hline fluor-18 & 1,83 & 634 & 194 & 511 & +++ & $350-600$ \\
\hline cuivre-64 & 12,7 & $578-653$ & 36 & 511 & +++ & $175-370$ \\
\hline \multirow[t]{2}{*}{ iode-124 } & 100,3 & $1532-2135$ & 46 & 511 & ++ & $74-158$ \\
\hline & & & 63 & 603 & & \\
\hline \multirow[t]{2}{*}{ yttrium-86 } & 14,7 & $\begin{array}{l}1221-1545- \\
1988-3141\end{array}$ & 64 & 511 & ++ & $185-370$ \\
\hline & & & 82 & 1077 & & \\
\hline \multirow[t]{3}{*}{ rubidium-82 } & 0,02 & 730 & 42 & 511 & ++ & 1850 \\
\hline & & & 62 & 555 & & \\
\hline & & & 84 & 777 & & \\
\hline fer-52 & 8,3 & $804-2633$ & 305 & 511 & + & \\
\hline \multicolumn{7}{|l|}{ Émetteurs $\beta^{-}$} \\
\hline yttrium-90 & 64 & 2284 & 0 & 0 & +++ & $1480-3700$ \\
\hline iode-131 & 192,7 & 606 & 82 & 365 & +++ & $3700-11000$ \\
\hline cuivre-67 & 61,9 & 390 & 49 & 185 & ++ & \\
\hline lutetium-177 & 161 & 497 & 11 & 208 & ++ & $3700-11000$ \\
\hline carbone-14 & 50194800 & 157 & 0 & & + & \\
\hline tritium & 107748 & 19 & 0 & & + & \\
\hline \multicolumn{7}{|l|}{ Émetteurs $\alpha$} \\
\hline bismuth-213 & 0,77 & 5869 & 28 & 440 & ++ & 3000 \\
\hline astate-211 & 7,2 & 5870 & 12 & 79 & + & $370-740$ \\
\hline actinium-225 & 240 & 5830 & 1 & 100 & + & \\
\hline radium-223 & 273,6 & 5871,3 & 14 & 269 & + & \\
\hline
\end{tabular}

dispositions en vigueur concernant l'aménagement d'un service de médecine nucléaire sont toujours celles définies dans l'arrêté du 30 octobre 1981 relatif aux conditions d'emploi des radioéléments artificiels utilisés en sources non scellées à des fins médicales. Ces dispositions ont été complétées au cours du temps, en particulier pour prendre en compte le développement du FDG. À cet effet, la notice DGSNR/SD9 L/04-V3 « Contraintes de radioprotection liées à l'utilisation 
de ${ }^{18}$ FDG dans une unité de médecine nucléaire » a été publiée par la DGSNR en 2005.

Parmi les dispositions relativement récentes, on peut également citer celles concernant les effluents et déchets radioactifs contenues dans la circulaire DGS/SD 7 D/DHOS/E $4 n^{\circ}$ 2001-323 du 9 juillet 2001 relative à la gestion des effluents et des déchets d'activités de soins contaminés par des radionucléides. Dans le cadre de cette circulaire, le texte « avis du ministère de la santé publique et de la sécurité sociale aux utilisateurs de radioéléments soumis au régime d'autorisation prévu par le code de la santé publique et relatif à l'élimination des déchets radioactifs», publié au JO du 6 juin 1970 est abrogé. Ces dispositions devraient être largement reprises dans la future réglementation. Il est intéressant de noter que l'essentiel des dispositions concerne le technétium-99m pour les applications diagnostiques et l'iode-131 en thérapie. Par exemple, une mesure de l'activité à l'émissaire ${ }^{3}$ de l'établissement doit conduire à des valeurs moyennes sur $8 \mathrm{~h}$ inférieures à $1000 \mathrm{~Bq} / \mathrm{L}$ pour le technétium-99m et $100 \mathrm{~Bq} / \mathrm{L}$ pour les autres radionucléides dont l'iode-131.

Nous allons aborder les trois aspects de la radioprotection liés aux évolutions en médecine nucléaire : le personnel, l'entourage et l'environnement.

\subsection{L'exposition du personnel du service de médecine nucléaire}

L'arrivée du fluor-18 a eu un impact essentiellement sur l'exposition externe et les moyens de protection adaptés au rayonnement de $511 \mathrm{keV}$, alors que l'utilisation de l'yttrium-90 pose surtout des problèmes vis-à-vis du bêta de $2284 \mathrm{MeV}$ et, à un degré moindre, vis-à-vis du rayonnement de freinage produit dans le patient.

\subsubsection{Cas du fluor-18}

Plusieurs études sont maintenant disponibles sur les niveaux d'exposition externe rencontrés lors d'un examen au FDG, depuis la préparation de la seringue jusqu'à la réalisation de l'examen. Les valeurs peuvent varier d'un auteur à l'autre compte tenu de l'activité injectée, des moyens de protection utilisés (protège seringue, écran), du mode d'injection voire même de la conception du service (ensemble de locaux dédiés au FDG ou locaux intégrés dans le service de médecine nucléaire). Une étude menée en 2003 lors de la mise en place de cette pratique clinique dans le service de médecine nucléaire de l'Institut Gustave-Roussy a conduit aux

3 Sortie du collecteur des eaux usées. 
résultats suivants (dose en $\mu \mathrm{Sv}$ ), pour une activité injectée de l'ordre de $500 \mathrm{MBq} /$ patient :

- exposition max. des extrémités :

- préparation de la seringue $\quad 430 \mu \mathrm{Sv}$ index gauche,

- injection

$184 \mu \mathrm{Sv}$ index droit,

- exposition max. « corps entier» :

- préparation de la seringue $\quad 8,5 \mu \mathrm{Sv}$,

- injection $9,1 \mu \mathrm{Sv}$,

- examen (mise en place) 2,2 $\quad 2 \mathrm{~Sv}$.

Il faut également noter que les débits de dose instantanés peuvent atteindre des niveaux relativement élevés, ces valeurs correspondent toujours à une injection de $500 \mathrm{MBq}$ de ${ }^{18} \mathrm{~F}$ et un examen une heure après l'injection :

- à $1 \mathrm{~m}$ du patient juste après l'injection

- derrière un paravent de protection, après l'injection

- lors de la mise en place du patient, 1 heure après injection, à environ 40-50 cm du patient

$$
\begin{aligned}
& \approx 100 \mu \mathrm{Sv} / \mathrm{h}, \\
& \approx 10 \mu \mathrm{Sv} / \mathrm{h},
\end{aligned}
$$

$\leq 140 \mu \mathrm{Sv} / \mathrm{h}$.

Ces valeurs doivent inciter à une vigilance accrue et à un strict respect du règlement intérieur dans les locaux concernés. On peut également souligner que l'association de plus en plus fréquente d'un scanner aux appareils de tomographie par positons requiert la conformité de l'installation vis-à-vis de la règlementation relative au scanner, mais il faut bien sûr adapter la nature et l'épaisseur des parois au rayonnement de $511 \mathrm{keV}$.

À titre de comparaison le tableau II indique le débit d'exposition (en $\mu \mathrm{Sv} / \mathrm{h})$ à $1 \mathrm{~m} \mathrm{du}$ patient pour quatre situations cliniques types : la scintigraphie osseuse après injection de $800 \mathrm{MBq}$ de ${ }^{99 \mathrm{~m}} \mathrm{Tc}$, la thérapie anti-cancéreuse avec $3700 \mathrm{MBq}$ de ${ }^{131} \mathrm{I}$ (cas du patient juste après administration thérapeutique d'iode-131),

\section{TABLEAU II}

Ordre de grandeur du débit d'exposition (en $\mu \mathrm{Sv} / \mathrm{h}$ ) à un mètre du patient dans 4 situations cliniques correspondant à l'utilisation de ${ }^{99 \mathrm{~m}} \mathrm{Tc},{ }^{131} \mathrm{I}$ et ${ }^{18} \mathrm{~F}$.

Rough estimate of exposure rates at a distance of 1 meter from the patient, in 4 clinical settings

\begin{tabular}{|c|c|c|}
\hline & $\begin{array}{l}\text { Activité } \\
\text { (MBq) }\end{array}$ & $\begin{array}{c}\text { Débit d'exposition à } 1 \mathrm{~m} \\
(\mu \mathrm{Sv} / \mathrm{h})\end{array}$ \\
\hline${ }^{99 \mathrm{~m}} \mathrm{Tc}$ (scinti osseuse) & 800 & 18 \\
\hline${ }^{131}$ I (thérapie anti-cancéreuse) & 3700 & 240 \\
\hline${ }^{131}$ I (traitement thyroïde) & 400 & 26 \\
\hline${ }^{18} \mathrm{~F}$ (examen TEP) & 500 & 80 \\
\hline
\end{tabular}
corresponding to ${ }^{99} \mathrm{~m} \mathrm{Tc},{ }^{131} \mathrm{I}$ and ${ }^{18} \mathrm{~F}$ uses. 
la thérapie cancéreuse ou non cancéreuse avec $400 \mathrm{MBq}$ (cas du patient passant une scintigraphie 3 jours après administration de $3,7 \mathrm{GBq}$ de ${ }^{131} \mathrm{I}$ ou après administration d'un activité thérapeutique dans les applications non cancéreuses) et l'examen TEP après injection de $500 \mathrm{MBq}$.

\subsubsection{Cas des émetteurs $\beta$ purs}

Lorsqu'un radionucléide émet à la fois des rayonnements bêtas et des photons $\left({ }^{131} \mathrm{I},{ }^{18} \mathrm{~F}, \ldots\right)$, on considère que si la protection est adaptée au rayonnement photonique elle est aussi efficace pour le rayonnement $\beta$. Par contre en présence d'un rayonnement bêta pur (le ${ }^{90} \mathrm{Y}$ par exemple), les comportements ne sont pas toujours adaptés. En effet l'habitude est de considérer que $10 \mathrm{~mm}$ de PMMA ${ }^{4}$ suffisent comme protection vis-à-vis de ce rayonnement. Si cela est vrai pour la plupart des émetteurs $\beta$ et en particulier ceux dont l'énergie maximale est inférieure à $2 \mathrm{MeV}$, cela est limite en pratique pour les énergie supérieures et, surtout, cela conduit à des protège seringues dont la dimension les rendent difficilement utilisables. Vis-à-vis des matériaux plus denses (plomb, tungstène, ...), on met en avant la production de rayonnement de freinage. Physiquement cela est correct mais on peut aussi penser que vis-à-vis du rayonnement photonique de freinage ce matériau lourd sera plus efficace. C'est ce que nous pouvons vérifier en comparant les débits de dose au contact de 2 protège seringues différents, l'un de $10 \mathrm{~mm}$ de PMMA et l'autre de $5 \mathrm{~mm}$ de tungstène, contenant une source de ${ }^{90} \mathrm{Y}$. Les débits de dose mesurés par thermoluminescence étaient respectivement de 2,4 et $1,7 \mu \mathrm{Sv} \mathrm{h}^{-1} \mathrm{MBq}^{-1}$.

En complément de cet aspect de radioprotection concernant les extrémités, il faut aussi s'interroger sur le niveau d'exposition après l'injection. En effet le patient constitue alors une source en raison du rayonnement de freinage. Le spectre de ce rayonnement est riche en basse énergie, aussi un tablier de protection en équivalent de plomb de $0,5 \mathrm{~mm}$ permet une atténuation d'un facteur $\approx 60$ conduisant à un débit d'exposition à environ $50 \mathrm{~cm} \mathrm{du}$ patient de l'ordre de quelques $\mu \mathrm{Sv} / \mathrm{h}$.

\subsection{L'exposition de l'entourage familial ou du personnel (hors service de médecine nucléaire)}

Règlementairement seuls les patients traités avec plus de $740 \mathrm{MBq}$ de ${ }^{131}$ I doivent être hospitalisés. Cette période dure de 3 à 4 jours pendant lesquels les visites sont limitées, voire même interdites. Il est à noter que cette durée varie d'un centre à

4 PMMA : poly methyl méthacrylate (encore appelé Plexiglas ${ }^{\mathrm{TM}}$ ). 
TABLEAU III

Ordre de grandeur du débit d'exposition à $1 \mathrm{~m}$ du patient lors de sa sortie du service de médecine nucléaire pour un certain nombre d'applications thérapeutiques et diagnostiques.

Rough estimate of exposure rates at a distance of 1 meter from the patient at release in several diagnostic and therapeutic applications.

\begin{tabular}{|c|c|c|c|c|c|}
\hline Examen & $\begin{array}{c}\text { Radio } \\
\text { nucléide }\end{array}$ & $\begin{array}{c}\text { Forme } \\
\text { chimique }\end{array}$ & $\begin{array}{l}\text { Activité } \\
\text { admin. } \\
(\mathrm{MBq})\end{array}$ & $\begin{array}{l}\text { Délai injection - } \\
\text { sortie } \\
\text { (h) }\end{array}$ & $\begin{array}{c}\text { Débit d'exposition* } \\
\text { à } 1 \text { m lors de la sortie } \\
\text { du service } \\
(\mu \mathrm{Sv} / \mathrm{h})\end{array}$ \\
\hline \multicolumn{6}{|l|}{ Thérapie } \\
\hline Ttt thyroïde & ${ }^{131} I$ & iodure & 3700 & 72 & 26 \\
\hline Ttt thyroïde & ${ }^{131} \mathrm{I}$ & iodure & 555 & 1 & 36 \\
\hline tumeurs & ${ }^{90} \mathrm{Y}$ & $\begin{array}{c}\text { analogue } \\
\text { somatostatine }\end{array}$ & 4440 & 24 & $\approx 4$ \\
\hline \multicolumn{6}{|l|}{ Diagnostic } \\
\hline squelette & ${ }^{99 \mathrm{~m}} \mathrm{Tc}$ & phosphonate & 600 & 4 & $\approx 7$ \\
\hline tumeurs & ${ }^{18} \mathrm{~F}$ & FDG & 400 & 2 & 30 \\
\hline
\end{tabular}

* prise en compte de la période physique uniquement.

l'autre et ne repose sur aucune base bien définie. Après ce délai, et pour toutes les autres applications de médecine nucléaire, le patient peut soit retourner chez lui, soit dans un autre établissement ou service d'hospitalisation. Il convient alors d'informer le patient ou son accompagnant des mesures de précaution à prendre pour limiter les risques d'exposition externe et de contamination. Cette information est exigée par l'article 1333-64 du code la santé publique et l'arrêté du 21 janvier 2004 relatif à l'information des personnes exposées aux rayonnements ionisants lors d'un acte de médecine nucléaire. Par contre, à ce jour, aucune disposition pratique n'est recommandée sur la base du débit d'exposition du patient ou de l'exposition de l'entourage. Il est vrai que les hypothèses d'exposition (temps, distance, situation, ...) sont tellement variables que toute estimation s'avère imprécise. L'approche la plus simple consisterait à s'appuyer sur le débit d'exposition à 1 mètre du patient à sa sortie. Le tableau III donne un aperçu de l'ordre de grandeur de ce débit pour un certain nombre de situations cliniques correspondant à des applications thérapeutiques et diagnostiques. Les valeurs ont été calculées en ne considérant que la décroissance physique, ce qui majore la valeur du débit dans la mesure où l'élimination biologique a été ignorée.

Une autre indication indirecte de l'exposition de l'entourage peut être donnée par le débit d'exposition (en $\mu \mathrm{Sv} / \mathrm{h}$ ) à 1 mètre pour une source de $1 \mathrm{MBq}$. Le tableau IV donne l'ordre de grandeur de ce débit pour les émetteurs $\gamma, \beta^{-}$et $\beta^{+}$. Les données concernant l'exposition externe sont extraites en particulier du guide 


\section{TABLEAU IV}

Débit d'exposition (en $\mu \mathrm{Sv} / \mathrm{h}$ ) à 1 mètre d'une source de $1 \mathrm{MBq}$ pour les radionucléides du tableau I (hors émetteurs $\alpha$ et $\beta^{-}$purs).

Dose rate (in $\mu \mathrm{Sv} / \mathrm{h}$ ) at a distance of one meter from a one $\mathrm{MBq}$ source for radionuclides in Table $I$ (excepted pure $\alpha$ and $\beta^{-}$emitters).

\begin{tabular}{|lcc|}
\hline Radionucléide & $\begin{array}{c}\text { Période } \\
\text { (heures) }\end{array}$ & $\begin{array}{c}\text { Débit d'exposition }(\mathbf{e n ~} \boldsymbol{\mu S v} / \mathbf{h}) \mathbf{a ̀} \\
\text { 1 mètre d'une source de } \mathbf{1} \text { MBq }\end{array}$ \\
\hline cuivre-64 & 12,7 & $3,6 \times 10^{-2}$ \\
fluor-18 & 1,83 & $1,88 \times 10^{-1}$ \\
\hline iode-124 & 100,3 & $2,05 \times 10^{-1}$ \\
\hline iode-131 & 192,7 & $7,65 \times 10^{-2}$ \\
\hline lutétium-177 & 161 & $7,64 \times 10^{-3}$ \\
\hline tritium & 107748 & s.o. \\
\hline carbone-14 & 50194800 & s.o. \\
\hline technétium-99m & 6 & $2,1 \times 10^{-2}$ \\
fer-52 & 8,3 & $4,7 \times 10^{-1}$ \\
rubidium-82 & 0,02 & $1,65 \times 10^{-1}$ \\
\hline yttrium-86 & 14,7 & $4,6 \times 10^{-1} *$ \\
\hline * estimation. & & \\
\hline
\end{tabular}

pratique Radioprotection \& Radionucléides (Delacroix et al., 2004). Pour les émetteurs $\alpha$ et $\beta^{-}$pur, cette valeur est sans objet en dehors du rayonnement de freinage essentiellement présent pour le ${ }^{90} \mathrm{Y}$ en raison de son énergie $\beta$ élevée $(\approx 2,3 \mathrm{MeV})$. Si on prend en compte la valeur du débit et la période physique, il apparaît qu'à activité identique le ${ }^{52} \mathrm{Fe}$, le ${ }^{86} \mathrm{Y}$ et le ${ }^{124} \mathrm{I}$ sont les radionucléides susceptibles de présenter le débit le plus élevé lorsque le patient quitte le service et pendant les heures qui suivent l'injection.

\subsection{L'exposition de l'environnement}

Les dispositions actuelles relatives à l'élimination des effluents radioactifs sont précisées dans la circulaire DGS/SD 7 D/DHOS/E $4 n^{\circ}$ 2001-323 du 9 juillet 2001 (relative à la gestion des effluents et des déchets d'activités de soins contaminés par des radionucléides). Il est prévu qu'une mesure de l'activité à l'émissaire de l'établissement soit effectuée régulièrement et les résultats des mesures seront comparés aux niveaux guides suivants :

- $1000 \mathrm{~Bq} / \mathrm{L}$ pour le technétium-99m ;

- $100 \mathrm{~Bq} / \mathrm{L}$ pour les autres radioéléments. 
L'estimation de l'impact dosimétrique pour une personne dont le poste de travail se trouverait à la sortie du collecteur d'eaux usées d'un établissement hospitalier a été effectuée en supposant que les effluents rejetés présentent en permanence les activités volumiques ci-dessus. L'exposition externe due au champ de rayonnements produit par les radionucléides contenus dans les eaux usées, et l'exposition interne consécutive à l'inhalation d'eau contaminée et d'aérosols, compte tenu d'une hypothèse de conditions de travail en atmosphère saturée, ont été considérées pour 2000 heures de travail annuel (40 heures $\times 50$ semaines) :

- vis-à-vis de l'exposition externe, pour une personne située à une distance d'un mètre de la surface de l'eau dans le cas le plus défavorable d'un mélange des deux radionucléides $\left({ }^{99 \mathrm{~m}} \mathrm{Tc}\right.$ et $\left.{ }^{131} \mathrm{I}\right)$, la dose reçue pour 2000 heures serait de $9 \mu \mathrm{Sv}$;

- vis-à-vis de l'exposition interne (eau inhalée + aérosols inhalés), pour une personne respirant $1,2 \mathrm{~m}^{3} \mathrm{~d}$ 'air/h, le volume total d'eau inhalée pour une année serait de $44 \mathrm{~L}$. D'où, pour un coefficient de dose efficace engagée par unité d'activité incorporée par inhalation de $7,4 \times 10^{-9} \mathrm{~Sv} / \mathrm{Bq}$ pour l'iode-131 (directive 96/29/Euratom, 1996), la dose engagée pour l'année serait de 32,5 $\mu \mathrm{Sv}$ (la dose pour le technétium serait de $0,9 \mu \mathrm{Sv}$ ).

Ainsi le bilan de l'exposition interne + externe s'élèverait à 41,5 $\mu \mathrm{Sv}$. Si on prend l'hypothèse que la personne passe $10 \%$ de son temps de travail à la sortie d'un collecteur hospitalier, la dose serait de l'ordre de $4 \mu \mathrm{Sv} / \mathrm{an}$.

En prenant les mêmes hypothèses pour les radionucléides cités dans le tableau IV (sauf le rubidium-82 en raison de sa courte période de 1,27 minute) et pour les émetteurs alpha listés dans le tableau I, on obtient une estimation de l'exposition externe et interne en considérant une concentration de $100 \mathrm{~Bq} / \mathrm{L}$ (Tab. V). Les valeurs de débit d'exposition à $1 \mathrm{~m}$ sont données par Delacroix et al. (2004) et les coefficients utilisés pour le calcul de l'exposition interne sont extraits du tableau B de la directive 96/29/Euratom (1996).

Ces hypothèses conduisent à des niveaux de dose calculés, en particulier pour les émetteurs alpha, qui sont sûrement très loin de refléter la réalité du risque associé. En effet l'utilisation de tous ces radionucléides, contrairement au ${ }^{99} \mathrm{~m}$ Tc et ${ }^{131}$ I largement employés au quotidien sur des centaines de patients, ne concernera que quelques personnes. Aussi il serait justifié d'étudier d'autres hypothèses que celle d'une concentration permanente de $100 \mathrm{~Bq} / \mathrm{L}$.

Plusieurs approches peuvent être envisagées pour évaluer le risque associé à ces nouveaux radionucléides. L'une d'elle serait de considérer les limites de concentration à ne pas dépasser pour les rejets d'effluents. Ces valeurs n'existant pas en France on peut tenter de les déduire à partir des coefficients de dose efficace 
TABLEAU V

Ordre de grandeur de l'exposition externe et interne liée à une concentration de $100 \mathrm{~Bq} / \mathrm{L}$ des effluents de l'établissement.

Rough estimate of external and internal exposure due to a $100 \mathrm{~Bq} / \mathrm{L}$ concentration in hospital effluent.

\begin{tabular}{|c|c|c|c|c|}
\hline & $\begin{array}{c}\text { Débit d'exposition } \\
\text { à } 1 \mathbf{~ m} \\
\left(\mu \mathrm{Sv} \mathrm{h}^{-1} \mathbf{B q}^{-1}\right)\end{array}$ & $\begin{array}{c}\text { Exposition } \\
\text { externe } \\
(\mu \mathrm{Sv})\end{array}$ & $\begin{array}{c}\text { Coefficient } \\
\text { inhalation } \\
\left(\mathrm{Sv} \mathrm{Bq} \mathbf{B q}^{-1}\right)\end{array}$ & $\begin{array}{c}\text { Exposition } \\
\text { interne } \\
(\mu \mathrm{Sv})\end{array}$ \\
\hline \multicolumn{5}{|c|}{ Émetteurs $\gamma, \beta^{-}$et $\beta^{+}$} \\
\hline tritium & 0 & 0 & $2,6 \times 10^{-10}$ & 1,1 \\
\hline carbone-14 & 0 & 0 & $5,8 \times 10^{-09}$ & 25,5 \\
\hline fluor-18 & $1,60 \times 10^{-07}$ & 5,1 & $5,9 \times 10^{-11}$ & 0,3 \\
\hline fer-52 & $4,70 \times 10^{-07}$ & 15 & $6,3 \times 10^{-10}$ & 2,8 \\
\hline cuivre-64 & $3,00 \times 10^{-08}$ & 1 & $1,2 \times 10^{-10}$ & 0,5 \\
\hline rubidium- 82 & s.o. & s.o. & s.o. & s.o. \\
\hline yttrium-86 & $4,60 \times 10^{-07}$ & 14,7 & $4,7 \times 10^{-10}$ & 2,1 \\
\hline technétium-99m* & $2,20 \times 10^{-08}$ & 0,7 & $1,2 \times 10^{-11}$ & 1 \\
\hline iode-124 & $1,70 \times 10^{-07}$ & 5,4 & $4,4 \times 10^{-09}$ & 19,4 \\
\hline iode-131 & $6,40 \times 10^{-08}$ & 2,1 & $7,4 \times 10^{-09}$ & 32,6 \\
\hline lutétium-177 & $7,00 \times 10^{-09}$ & 0,2 & $1,2 \times 10^{-09}$ & 5,3 \\
\hline \multicolumn{5}{|l|}{ Émetteurs $\alpha$} \\
\hline astate-211 & - & - & $1,1 \times 10^{-07}$ & 484 \\
\hline bismuth-213 & - & - & $3,0 \times 10^{-08}$ & 132 \\
\hline radium-223 & - & - & $8,7 \times 10^{-06}$ & 38280 \\
\hline actinium-225 & - & - & $8,5 \times 10^{-06}$ & 37400 \\
\hline
\end{tabular}

* pour $1000 \mathrm{~Bq} / \mathrm{L}$.

engagée par unité incorporée par ingestion $(\mathrm{Sv} / \mathrm{Bq})$ pour la population (tableau $\mathrm{A}$ de la directive 96/29/Euratom, 1996). Ces coefficients permettent de connaître le nombre de becquerels correspondant à $1 \mathrm{mSv}$, limite annuelle d'exposition pour la population. En divisant cette valeur par 730 litres, qui est considérée comme la consommation d'eau annuelle moyenne de l'homme de référence, on obtient la limite en $\mathrm{Bq} / \mathrm{L}$ de la concentration radioactive des effluents à ne pas dépasser (Tab. VI). Cette approche est basée sur l'hypothèse que les effluents sont assimilables à de l'eau potable. On en voit donc les limites et la nécessité d'introduire des facteurs de décroissance et de dilution pour tenir compte du devenir de ces effluents entre le moment du rejet à la sortie de l'établissement et la sortie de la station de traitement. 
TABLEAU VI

Estimation des concentrations des effluents radioactifs en $\mathrm{Bq} / \mathrm{L}$ conduisant à une exposition de $1 \mathrm{mSv} / a n$ pouvant être retenues d'après les données de la directive EURATOM 96/29 (1996).

Rough estimate of radioactive waste concentration $(\mathrm{Bq} / \mathrm{L})$ resulting in an exposure of $1 \mathrm{mSv} / \mathrm{year}$. Values calculated from data in European directive 96/29 (1996).

\begin{tabular}{lcc}
\hline & Période (heures) & Concentration des effluents $(\mathbf{B q} / \mathbf{L})$ \\
\hline Émetteurs $\gamma, \beta^{-}$et $\beta^{+}$ & & \\
\hline tritium & 107748 & $7,6 \times 10^{4}$ \\
\hline carbone-14 & 504800 & $2,4 \times 10^{3}$ \\
\hline fluor-18 & 1,83 & $2,8 \times 10^{4}$ \\
\hline fer-52 & 8,3 & $9,8 \times 10^{2}$ \\
\hline cuivre-64 & 12,7 & $1,1 \times 10^{4}$ \\
\hline rubidium-82 & 0,02 & 5.0. \\
\hline yttrium-86 & 14,7 & $1,4 \times 10^{3}$ \\
\hline technétium-99m & $\mathbf{6}$ & $\mathbf{6 , 2} \times \mathbf{1 0 ^ { 4 }}$ \\
\hline iode-124 & 100,3 & $1,1 \times 10^{2}$ \\
\hline iode-131 & $\mathbf{1 9 2 , 7}$ & $\mathbf{6 , 2} \times \mathbf{1 0}$ \\
\hline lutétium-177 & 161 & $2,6 \times 10^{3}$ \\
\hline Émetteurs $\alpha$ & 243,6 & $1,4 \times 10^{1}$ \\
\hline astate-211 & 0,77 & $5,7 \times 10^{1}$ \\
\hline bismuth-213 & 7,2 & $1,3 \times 10^{2}$ \\
\hline radium-223 & 240 & $6,9 \times 10^{3}$ \\
\hline actinium-225 & & \\
\hline
\end{tabular}

Une autre approche serait de se baser sur les éléments figurant dans la réglementation relative aux eaux potables (décret 2001-1220, 2001 ; arrêté du 12 mai, 2004). On y relève la notion de dose totale indicative (DTI), qui doit être inférieure à $0,1 \mathrm{mSv}$, ainsi que des valeurs guides de $0,1 \mathrm{~Bq} / \mathrm{L}$ et $1 \mathrm{~Bq} / \mathrm{L}$ respectivement pour une activité alpha globale et bêta globale.

Une DTI de 0,1 mSv conduit en pratique aux valeurs du tableau VI divisées par 10. Mais dans la mesure où on se trouve en présence d'un mélange de radionucléides il faudrait pondérer ces concentrations en fonction du pourcentage respectif moyen de chacun d'entre eux.

À titre de comparaison, les valeurs concernant le ${ }^{99 \mathrm{~m}} \mathrm{Tc}$ et le ${ }^{131}$ I ont également été portées dans le tableau VI. Rappelons qu'actuellement les dispositions réglementaires en France fixent des valeurs guides, respectivement de 1000 Bq/L 
et de $100 \mathrm{~Bq} / \mathrm{L}$ pour ces deux radionucléides. On constate que la valeur retenue pour le ${ }^{99 \mathrm{~m}} \mathrm{Tc}$ est très en dessous de la valeur déduite de notre calcul, quelle que soit l'approche retenue, alors que pour le ${ }^{131}$ I on est plus dans le cas de la première approche que de celle basée sur la réglementation de l'eau potable. Il est à noter qu'une réflexion globale devrait tenir compte de l'exposition externe associée pour les émetteurs gamma.

Cette analyse n'a pas pour objectif de proposer des valeurs de concentration des effluents radioactifs mais de montrer la nécessité de procéder à des études d'impact pour connaître plus précisément l'effet réel des rejets hospitaliers, non pas sur la base de valeurs de contrôle, mais sur celle de la nature des radiopharmaceutiques utilisés, du nombre d'actes effectués et du nombre de patients hospitalisés ou traités en ambulatoire.

\section{Discussion et conclusion}

Les évolutions récentes et futures des applications diagnostiques et thérapeutiques en médecine nucléaire sont très prometteuses d'un point de vue clinique. Cependant les caractéristiques physiques de bon nombre de ces nouveaux radionucléides doivent inciter les utilisateurs, ainsi que les responsables concernés, à analyser tous les aspects de radioprotection liés à ces nouvelles pratiques.

L'utilisation de sources radioactives sous forme non scellée et leur administration à un patient font que ces aspects de radioprotection se posent non seulement lors de la préparation et de l'administration du radiopharmaceutique, mais également au niveau du patient pendant son séjour dans le service puis à l'extérieur et enfin vis-à-vis de l'environnement.

Les données récentes concernant le ${ }^{18} \mathrm{~F}$ et le ${ }^{90} \mathrm{Y}$, montrent que les moyens et méthodes de radioprotection doivent faire l'objet d'une réflexion non pas lors de la mise en utilisation clinique de ces radionucléides mais plus en amont, dès la phase de développement. L'extension de notre réflexion aux futurs radiopharmaceutiques potentiellement utilisables chez l'homme montre qu'une attention particulière doit être portée sur 2 points :

- Sur quelle base définir les consignes de confinement des malades (Quand ? Combien de temps ?) et quelles consignes lors de la sortie du service de médecine nucléaire?

- Quel modèle de calcul appliquer et quelle concentration radioactive accepter par les effluents radioactifs, surtout ceux comportant émetteurs alpha ? 
Pour répondre à ce dernier point, il serait sûrement intéressant d'étudier la possibilité de mener une démarche similaire à celle pratiquée pour les rejets des installations nucléaires et de l'extrapoler au milieu médical.

\section{RÉFÉRENCES}

Arrêté du 12 mai (2004) fixant les modalités de contrôle de la qualité radiologique des eaux destinées à la consommation humaine.

Couturier O., Luxen A., Chatal J.F., Vuillez J.P., Rigo P., Hustinx R. (2004) Fluorinated tracers for imaging cancer with positron emission tomography, Eur. J. Nucl. Med. Mol. Imag. 31, 11821206.

Décret no. 2001-1220 (2001) du 20 décembre 2001 relatif aux eaux destinées à la consommation humaine, à l'exclusion des eaux minérales naturelles, JO du 20 décembre 2001.

Delacroix D., Guerre J.P., Leblanc P. (2004) Guide pratique Radioprotection \& Radionucléides. EDP Sciences, Les Ulis.

Directive Euratom 96/29 (1996) du conseil du 13 mai 1996 fixant les normes de base relatives à la protection sanitaire de la population et des travailleurs contre les dangers résultant des rayonnements ionisants.

Gotthardt M., Boermann O.C., Behr T.M., Behe M.P., Oyen W.J. (2004) Development and clinical application of peptide-based radiopharmaceuticals, Curr. Pharm. Des. 10, 2951-2963.

Machac J. (2005) Cardiac positron emission tomography imaging, Semin. Nucl. Med. 35, 17-36.

Robinson M.K., Doss M., Shaller C., Narayanan D., Marks J.D., Adler L.P., Gonzalez Trotter D.E., Adams G.P. (2005) Quantitative immuno-positron emission tomography imaging of HER2positive tumor xenografts with an iodine-124 labeled anti-HER2 diabody, Cancer Res. 65, 1471-1478.

Sharkey R.M., Goldenberg D.M. (2005) Perspectives on cancer therapy with radiolabeled monoclonal antibodies, J. Nucl. Med. 46 (Suppl 1), 115S-127S. 